\title{
Comparing Physician and Patient Perspectives on Prophylactic Treatment with BAY 94-9027 for Severe Haemophilia A: A Post Hoc Analysis
}

\author{
Shadan Lalezari · Michaël Acquadro · Elodie de Bock · \\ Jérémy Lambert · Mindy L. Simpson
}

Received: March 13, 2020 / Published online: May 14, 2020

(C) The Author(s) 2020

\begin{abstract}
Introduction: BAY 94-9027 is a newly developed extended half-life product to treat haemophilia, allowing for fewer injections than with standard products. This post hoc analysis aimed to compare physicians' and patients' opinions on BAY 94-9027 prophylaxis, and explore how qualitative interview data is aligned with the data from the Haemophiliaspecific Quality of Life questionnaire for Adults (Haemo-QoL-A).

Methods: Exploratory qualitative interviews were conducted with physicians and patients by phone upon the exit of patients from the PROTECT VIII extension phase following a semidirected guide. In this post hoc analysis, all transcripts were reviewed and reported concepts were compared to assess the level of concordance
\end{abstract}

Digital Features To view digital features for this article go to https://doi.org/10.6084/m9.figshare.12221039.

\section{S. Lalezari}

National Hemophilia Center, Sheba Medical Center, Tel Hashomer, and Sackler School of Medicine,

Tel-Aviv University, Tel-Aviv, Israel

M. Acquadro $\cdot$ E. de Bock $\cdot$ J. Lambert

Patient-Centered Outcomes, ICON plc, Lyon, France

\section{L. Simpson ( $\square)$}

Rush Hemophilia and Thrombophilia Center, Rush University Medical Center, Chicago, IL, USA

e-mail: mindy_1_simpson@rush.edu between physicians and patients. These qualitative data were compared with the Haemo-QoL-A mean global and subscale scores at baseline and end of main phase (36 weeks later).

Results: Ten physicians and 16 patients (mean age 47 years) from Israel, the Netherlands and the USA were interviewed. Significant improvements were reported by all physicians from baseline [e.g. lower frequency of bleeds (80\%), improvement in emotional functioning (90\%)], which is in concordance with patients' reports. The improved confidence reported by physicians cascaded to greater participation in various activities, resulting in a better perceived emotional state and a significant improvement on the Haemo-QoL-A emotional impact subscale score $(p=0.04)$ between baseline and end of main phase. Most physicians $(80 \%)$ reported improvement in bleed frequency, as patients did (88\%). Improvement in physical functioning or mobility was not consistently reported in this 8-month study.

Conclusion: Interviewed physicians and patients generally agreed on the beneficial impact of BAY 94-9027, specifically regarding the increased level of self-confidence in patients and its subsequent positive impact on patients' lives. These findings supported the observed improvement on the Haemo-QoL-A emotional impact subscale. Overall, this study highlights the concordance between physician and patient perspective on the positive experience with BAY 94-9027. 
Keywords: Haemophilia A; Patient-reported outcome measures; Qualitative research; Quality of life

\section{Key Summary Points}

Why carry out this study?

The efficacy and safety of BAY 94-9027, administered as on-demand or prophylactically with dosing regimens from two times per week up to every 7 days, was demonstrated in the pivotal phase $2 / 3$ PROTECT VIII study in previously treated patients with severe haemophilia A.

It is key to include patient voice into drug development.

The experience and the impact of BAY 949027 on patients' quality of life has been previously reported, but a question remains on how it aligns with physician perspective.

\section{What was learned from the study?}

Physicians' positive description of patients' experience with BAY 94-9027 generally agrees with patient-reported experience.

Qualitative findings from the interviews supported the observed quantitative improvement in the Haemo-QoL-A emotional impact subscale.

\section{INTRODUCTION}

Haemophilia A is a chronic disease producing physical disability and, if not adequately managed at an early age, can be potentially lifethreatening. Early treatment can also prevent future joint disease and/or damage [1]. It is an inherited deficiency of the clotting factor VIII (FVIII) caused by mutations in the factor VIII gene on the $\mathrm{X}$ chromosome. Severe

haemophilia A is characterised clinically by recurrent spontaneous or traumatic bleeds into soft tissues and joints, the latter leading to progressive and irreversible joint damage [1]. Haemophilia A occurs worldwide with an estimated incidence of 1 in 5000 male births [2]. The current standard of care aims to replace the missing clotting factor by intravenously administering a factor concentrate either episodically as bleeds occur (on-demand regimen) or on a preventive regular basis (prophylaxis regimen) [1]. The half-life of standard available recombinant FVIII (rFVIII) products is limited to $8-12 \mathrm{~h}$ [3]. The high frequency of infusion of current treatment regimens required to maintain protective FVIII levels in the blood and related venous access problems can be a barrier to prophylaxis adherence and are burdensome to individuals with haemophilia $[4,5]$.

BAY 94-9027 (damoctocog alfa pegol) is a PEGylated B-domain-deleted rFVIII [6]. Deletion of the $\mathrm{B}$ domain does not result in any loss of function but allows for site-specific PEGylation while preserving its interaction with von Willebrand factor (factor VIII carrier) and its coagulation activity $[6,7]$. The PEGylation helps slow hepatic clearance, leading to an enhanced pharmacokinetic profile $[8,9]$. The enhanced pharmacokinetic profile of BAY 94-9027 translates in turn into higher and sustained FVIII levels over time, as per head-tohead pharmacokinetics comparisons against other products [10]. As this article was developed, BAY 94-9027 (Jivi) as well as other FVIII extended half-life products have received regulatory approval in several regions of the world.

The efficacy and safety of BAY 94-9027, administered as on-demand or prophylactically with dosing regimens from two times per week up to every 7 days, was demonstrated in the pivotal phase 2/3 PROTECT VIII study in previously treated patients with severe haemophilia A (ClinicalTrials.gov identifier NCT01580293) [11]. For the assessment of the long-term efficacy and safety of BAY 94-9027, subjects were given the possibility at the end of the main PROTECT VIII to roll over into an optional extension study of at least 6 months [12].

To better capture subject experience and the impact of BAY 94-9027 on patients' health-re- 
lated quality of life (HRQoL), participants completed patient-reported outcome (PRO) instruments, including the Haemophilia-specific Quality of Life questionnaire for Adults (HaemoQoL-A) during the main study. In addition, physicians and study participants from the investigator sites in Israel, the Netherlands and the USA were invited to participate in qualitative exploratory interviews when exiting the extension part of the study. The findings from the patient interviews were recently published and showed that participants reported that BAY 949027 received during the PROTECT VIII study resulted in their increased ability to participate in physical activities, better 'vein health', less time needed to schedule and administer factor VIII, reduced impact on work and improved emotional well-being [13].

The objective of this post hoc analysis was to compare physicians' and patients' perspectives in the qualitative data source, as well as further examining the patient experience by linking qualitative and quantitative data.

\section{METHODS}

\section{Study Design}

PROTECT VIII was a multinational, partially randomised, open-label trial evaluating the safety and efficacy of BAY 94-9027 with different dosing frequencies in both the prophylactic and on-demand treatment of bleeding in subjects who were 12 years of age and older with severe haemophilia A ( $<1 \%$ FVIII) and who had a documented history of at least 150 exposure days with any FVIII product. The total duration of the main trial was approximately 36 weeks after which subjects could enter an extension study $[11,12]$.

\section{Health-Related Quality of Life Assessments}

The HRQoL of patients was captured at baseline and also at the end of the main study (week 36) using the self-administered validated HaemoQoL-A [14]. The questionnaire encompasses 41 items that cover six domains: Physical functioning, Role functioning, Worry, Consequences of bleeding, Emotional impact, and Treatment concerns. The domain scores range from 0 to 100 , with higher scores meaning better HRQoL or less impairment. A global score can be derived by calculating the mean of all subscale scores.

\section{Physician and Patient Interviews}

Optional qualitative exploratory interviews were conducted to assess treatment satisfaction and the HRQoL of subjects treated with BAY 949027 in the PROTECT VIII study compared to prior therapy. The perspective taken was that of the treating physicians as well as their patients from three countries (Israel, Netherlands and USA) as patients exited the extension study. Physicians involved in the recruitment for the patient interviews and had also treated adult patients with BAY 94-9027 during the clinical study were invited to participate in optional phone interviews. In parallel, adult study patients who received BAY 94-9027 as a prophylactic treatment were invited to participate in optional phone interviews.

More details around the recruitment method and the conduct of the interviews with the patients has been published elsewhere [13].

Briefly, all physicians were provided with information and personally signed and dated a legally effective agreement before participating in the interviews. Patients and physicians were interviewed over the same time period. All patients were provided with written information about the study from their physician and written informed consent was obtained by their physician before their participation in any interview-related activities. Both patient and physician interviews followed dedicated interview guides, to be used following a semi-directive technique, to collect information about the patient experience with their disease, the previous haemophilia treatment they had received prior to the PROTECT VIII study, and the difference with BAY 94-9027. The semi-directive technique involves open-ended questions in order to collect spontaneously reported information. If themes are not spontaneously 
addressed by the patient or clinician, the interviewer will specifically revive these themes, as indicated in the interview guide [15].

Interviews were audio-recorded and transcribed verbatim and de-identified. Briefly, patients were eligible if they had been randomised in one of the prophylactic arms in PROTECT VIII and further rolled over in the extension study, and if they had personally signed and dated an informed consent. Patients were not eligible if not able or willing to complete a 1-h interview. Physicians were eligible if they had at any point during PROTECT VIII and its extension managed and/or treated adult patients with BAY 94-9027.

\section{Post Hoc Analysis}

\section{Comparing Physician and Patient Interviews}

The interview transcripts were reviewed as well as the list of codes used to tag concepts raised during the interviews. Transcripts had been coded using a thematic analysis process on ATLAS.ti, dedicated software facilitating qualitative analysis [16]. General themes were identified and then common concepts that physicians and patients evoked during the interviews were compared to assess concordance between physicians' and patients' reports inferentially. Physicians' and patients' extracted themes were tracked along with verbatim illustrations from the interview transcripts. Analysis of physician and patient interviews occurred simultaneously, both contributing thematic analysis.

\section{Comparing Quantitative Results with Qualitative Results}

The Haemo-QoL-A domain scores and global score of the interviewed patient population at baseline and at the end of the main study (week 36) were compared to the qualitative data extracted in the interviews. The significance of the change in score was assessed using a paired $t$ test for each score.

\section{Compliance with Ethics Guidelines}

The study was performed in accordance with the Helsinki Declaration of 1964, and its later amendments. Relevant ethical approvals were sought at the central and/or local level, as required by each recruiting clinical site. This included approvals for the conduct of this study from the following committees: Sheba Medical Center Independent Review Board (Israel), Committee of Advice nWMO-Medical Ethical Committee at Universitair Medish Centrum Groningen (Netherlands), Hagaziekenhuis local ethics committee (Netherlands), Maastricht University Medical Center-Clinical Trial Center Maastricht (CTCM) local ethics committee (Netherlands) and the Copernicus Group Independent Review Board (USA). Patients had personally signed and dated an informed consent document prior to being interviewed.

\section{RESULTS}

\section{Interviewed Population}

In total, 10 physicians and 16 patients were interviewed between May and September 2017. Among the interviewed physicians, two were from Israel, four from the Netherlands and four from the USA. They had between 5 and 25 years of experience in treating patients with haemophilia and were seeing on average 5-40 patients with haemophilia per month.

All patients who participated in the interviews were on prophylactic regimen with different frequencies of injections. Most patients were located in Israel $(n=9)$, followed by the Netherlands $(n=5)$ and USA $(n=2)$. More than one physician could manage the same trial participant over the course of the trial and its extension, explaining the discrepancy between the number of physicians and patients interviewed in the USA. Mean age of the patients was 47 years. The majority of the participants (69\%) were receiving BAY 94-9027 treatment once every 5 days. At the time of the interview, participants had been receiving BAY 94-9027 for a mean duration of 57 months. Patients' characteristics are summarised in Table 1. 
Table 1 Interviewed patients' characteristics as reported by patients $(n=16)$

\begin{tabular}{|c|c|}
\hline Characteristic & Value \\
\hline Mean age (range) at time of interview, years & $46.6(29-68)$ \\
\hline \multicolumn{2}{|l|}{ Work status, $n(\%)$} \\
\hline Working full or part time & $11(68.8)$ \\
\hline Looking for work & $3(18.8)$ \\
\hline Retired & $1(6.3)$ \\
\hline Missing data & $1(6.3)$ \\
\hline Mean time (range) since diagnosis, years & $44.6(27-64)$ \\
\hline Mean time (range) since starting therapy, years & $40.4(3-68)$ \\
\hline Mean number (range) of bleeds during last 12 months before enrolment & $3.81(0-25)$ \\
\hline Mean number (range) of joint bleeds during last 12 months before enrolment & $3.19(0-23)$ \\
\hline \multicolumn{2}{|l|}{ Previous factor VIII (multiple factor possible), $n$ (\%) } \\
\hline Kogenate/Helixate FS & $5(31.3)$ \\
\hline Advate & $1(6.3)$ \\
\hline ReFacto AF/Xyntha & $1(6.3)$ \\
\hline Factor VIII recombinant/not specified & $10(62.5)$ \\
\hline \multicolumn{2}{|l|}{ Previous treatment regimen, $n(\%)$} \\
\hline Prophylaxis & $16(100)$ \\
\hline Mean duration (range) of BAY $94-9027$ treatment, months ${ }^{\mathrm{a}}$ & $56.7(52-66)$ \\
\hline \multicolumn{2}{|l|}{ Treating with BAY 94-9027 at time of interview, $n$ (\%) } \\
\hline Yes & $13(81.3)$ \\
\hline No & $3(18.8)$ \\
\hline \multicolumn{2}{|l|}{ Frequency of BAY 94-9027 administration at time of interview, $n$ (\%) } \\
\hline 2 times a week & $2(12.5)$ \\
\hline Every 5 days & $11(68.8)$ \\
\hline Every 7 days & $3(18.8)$ \\
\hline
\end{tabular}

${ }^{a}$ Last 12 months from the interview; note that at time of interview, the majority of patients (13/16) were still treated with BAY 94-9027 in the extension phase

\section{Physician and Patient Perspectives on BAY 94-9027}

Several themes were identified in both the physician and the patient interviews. Those themes were grouped into four broad categories: treatment-related attributes, physical functioning, emotional/psychological functioning, and social and work functioning. More details about each theme are provided below and illustrative quotes are available in Table 2. 
Table 2 Themes identified in both physician and patient interviews and illustrative quotes

\begin{tabular}{|c|c|}
\hline Categories and themes & Quotes \\
\hline \multicolumn{2}{|l|}{ Treatment-related attributes } \\
\hline Infusion frequency & $\begin{array}{l}\text { "You know, it is nicer-you don't have to think about it as much. Just once a week. So, I } \\
\text { mean it definitely gave me a little bit more time to stay in my routine and get things } \\
\text { done... Being able to do more things." (US-02 patient infused every } 7 \text { days) } \\
\text { "What are the most important things to you in a treatment for hemophilia? Less impact on } \\
\text { my body is nice. So, the frequency helps out, I only have to stick once a week." (US-02 } \\
\text { patient infused every } 7 \text { days) }\end{array}$ \\
\hline $\begin{array}{l}\text { Pain comparison with new } \\
\text { treatment }\end{array}$ & $\begin{array}{l}\text { "Every bleed is accompanied by pain. Was the pain as bad as in the past? The intensity of } \\
\text { the pain varies with the severity of the bleed. If the bleed is serious, the pain is very strong. } \\
\text { Relative to the previous treatment, was the pain just as bad? There is no difference in the } \\
\text { level of pain." (Israeli-05 patient infused every } 5 \text { days) } \\
\text { "With the fact that you have to inject less frequently. Not that it hurts to do it, but when } \\
\text { you get older, it is more difficult to find a good vein, you have to search a bit and then it is } \\
\text { pleasant that you do not have to do this so often... but it is not really that much of a deal. } \\
\text { You just have to do your best and then you are done for } 5 \text { days." (Dutch-02 patient } \\
\text { infused every } 5 \text { days) }\end{array}$ \\
\hline Efficacy & $\begin{array}{l}\text { "Once every five days I had a bleed, but as soon as I switched to twice a week I had bleeds } \\
\text { three or four times a year, and they weren't serious." (Israeli-01 patient infused two times } \\
\text { a week) }\end{array}$ \\
\hline Adherence & $\begin{array}{l}\text { "All of my patients report to me that they are more likely to take a drug that they have to } \\
\text { take less, less often with, with regards to infusional drugs like hemophilia drugs." (US-01 } \\
\text { physician) }\end{array}$ \\
\hline Physical functioning & $\begin{array}{l}\text { "How does [BAY 94-9027] make you feel, physically? Truthfully, I don't think that can be } \\
\text { changed. It's already a given" (Israeli-05 patient infused every } 5 \text { days) } \\
\text { "Listen, I walk. I go down stairs. I'm not afraid of having a bleed. The factor lasts in my } \\
\text { body." (Israeli-06 patient infused every } 7 \text { days) } \\
\text { "So the restrictions are not because of bleeds, but because of joint erosion? Yes." (Israeli-04 } \\
\text { patient infused every } 5 \text { days) }\end{array}$ \\
\hline
\end{tabular}


Table 2 continued

\begin{tabular}{|c|c|}
\hline Categories and themes & Quotes \\
\hline $\begin{array}{l}\text { Emotional/psychological functioning } \\
\text { (worry, confidence) }\end{array}$ & $\begin{array}{l}\text { "There are patients that find the administering of coagulation factors } \\
\text { confrontational, because it confronts them with the fact that they have a disease, } \\
\text { and then it has an emotional impact." (Dutch- } 02 \text { physician) } \\
\text { "I am less fearful. Since the drug is in my body for longer, I don't worry so much } \\
\text { about when I last injected or how much. So I worry less." (Israeli-07 patient } \\
\text { infused every } 5 \text { days) } \\
\text { "I was taking one-third less injections and I felt safer that I was covered. The period } \\
\text { of the study was better for me, psychologically speaking. One-third fewer } \\
\text { injections is significant." (Israeli-01 patient infused two times a week) } \\
\text { "They have a lot of confidence in that they are protected from bleeding, um, and so } \\
\text { they're able to go about their usual activities, you know, confidently and feeling } \\
\text { like they're, they're safe to participate because they, you know, then they know } \\
\text { they're not going to bleed." (US-04 physician) }\end{array}$ \\
\hline \multicolumn{2}{|l|}{ Social and work functioning } \\
\hline Social activities & $\begin{array}{l}\text { "From the confidence that I have factor that will last } 5 \text { days, and it lasts longer in } \\
\text { my body. You have more confidence. You don't worry as much about going out } \\
\text { for a meal with friends, for example, about when you last took factor and if a } \\
\text { spontaneous bleed will ruin your evening." (Israeli-0 } 5 \text { patient infused every } \\
5 \text { days) }\end{array}$ \\
\hline Work & $\begin{array}{l}\text { "It's still management but even just walking around the job site was difficult. I took } \\
\text { crutches with me wherever I went pretty much. That or a golf club used as a } \\
\text { cane." (US-02 patient infused every } 7 \text { days) }\end{array}$ \\
\hline
\end{tabular}

\section{Treatment-Related Attributes}

All physicians discussed the general impact of frequency of infusions; six mentioned that time to prepare/administer was burdensome and almost all physicians (80\%) mentioned that higher frequency was linked to higher burden. Thirteen patients out of 16 discussed this topic; five patients found that time to infuse the medication was not problematic and was part of routine while two mentioned that this was problematic. Nine patients mentioned infusion frequency to be easier to manage with BAY 949027. Nine physicians reported that infusion frequency was very important, two physicians added that it was now important because it was possible to act on the infusion frequency with the availability of extended half-life products. Ten patients reported infusion frequency to be the most important aspect when talking about treatment satisfaction, two patients reported that it was of moderate importance while three patients reported that it was of low importance. Overall, there was some concordance about both the importance of the frequency of infusions and the related burden, as it requires time and planning.

Three physicians discussed symptoms and compared pain with the new treatment to the previous treatment; two mentioned that the treatment becomes less painful over time, and 
that there is a reduction in chronic pain related to fewer bleeds, while one physician mentioned that fewer injections were a less negative experience for patients. Fourteen patients out of 16 discussed this topic, seven patients found that intensity of pain related to bleeding was the same whilst two mentioned that it was less than before. Four patients mentioned that the pain related to injection was less frequent than before. Overall, there was concordance about pain related to injection: the pain was less frequent if there were fewer injections. Pain regarding bleeding was considered to be more or less the same.

Eight physicians discussed the physical impact of fewer injections; seven reported a benefit on their patients' vein health, while one physician reported that there was no change in his/her patient quality of life. Fourteen patients out of 16 discussed this topic; ten patients reported that they found a benefit in minimising acute/long-term damage on their veins, three patients reported that their veins could heal between injections, while four patients reported little to no benefits. Overall, there was concordance regarding benefits to patients' vein health.

Eight physicians discussed the efficacy of BAY 94-9027 and especially the frequency of bleeds; seven reported that it was lower, and one physician reported that their patient was not bleeding anymore. All patients discussed this topic; 14 patients reported that it was lower, while two patients reported that it was the same. One physician discussed also the severity of bleeds, and he said that the severity was lower. Nine patients discussed this topic; four patients reported that it was lower, while five patients reported that it was the same. Overall, there was concordance regarding lower frequency of bleeds for patients, and that the severity of bleeds did not change and was the same as before.

Eight physicians discussed adherence to BAY 94-9027; five physicians reported that their patients were generally adherent or very adherent with their previous treatments, but four of these clinicians reported an improvement of patients' adherence to BAY 94-9027 compared to other treatments. Three patients out of 16 discussed this topic; three patients reported that it was easier to adhere to it compared to other treatments. Overall, there was concordance regarding adherence to BAY 949027, it was easier than other treatments.

All physicians discussed the mode of administration of treatments for haemophilia; ten reported that patients had an aversion toward injections, four physicians said that patients would rather have another means of administration, and two physicians reported that their patients would rather have a subcutaneous treatment. All patients discussed this topic; all patients reported that they had an aversion or were neutral toward injections. Four patients discussed that they would rather take a pill, and one patient said that he/she would rather have subcutaneous treatment. Overall, there was concordance regarding the mode of administration: patients would prefer something different than intravenous injections.

Eight physicians discussed the drawbacks of the new treatment; four reported that the new treatment would be expensive, two discussed that patients were less confident with fewer injections at onset, one said that this would induce a lack of compliance because the treatment is less frequent, one reported that it was still too frequent and that intravenous injections were not good. Seven patients out of 16 discussed this topic; one patient reported that the new treatment was expensive, one patient said that it had side effects, one patient reported that the onset of healing was not as fast as other treatments and one patient reported that the number of injections was still too frequent (twice a week), and three patients said that there were no drawbacks. Overall, there was no real concordance regarding the treatment's drawbacks. Last, six physicians and 11 patients spontaneously reported they would recommend the use of BAY 94-9027 for the treatment of haemophilia A.

\section{Physical Functioning and Participation in Physical Activities}

Four physicians discussed the physical functioning with BAY 94-9027; three mentioned that there was no change in the physical function as reported by patients. One physician 
mentioned that there was potential improvement, whilst three reported that there was no potential improvement with more factor product. Seven patients out of 16 patients discussed this topic; six found that there was no change in their physical function whilst one mentioned that he still had problems walking. Overall, there was high concordance regarding physical function as patients and physicians reported that no change was experienced.

Nine physicians discussed physical activity following treatment with BAY 94-9027; four mentioned that there was an improvement in participation in physical activities whilst five of them mentioned that there was no real improvement. Thirteen patients out of the 16 discussed this topic; nine patients found that there was an improvement in participation in physical activities whilst four did not find an improvement. Nine patients mentioned that they still had some restrictions when they wanted to participate in physical activities. Overall, patients were more optimistic than physicians regarding participation in physical activities: patients were able to participate in more various physical activities than before owing to their improved confidence, despite reports that the improvements provided by the treatment in terms of bleed frequency were mitigated as a result of existing restrictions such as damaged joints.

\section{Emotional/Psychological Functioning}

Eight physicians discussed the emotional and psychological burden of haemophilia; all of them mentioned the emotional impact of living with this chronic disease. Nine patients out of 16 discussed this topic; three patients found that having an ongoing treatment was a necessity for them to be reassured. Two patients mentioned a fear of developing antibodies, two patients discussed their anxiety regarding duration of treatment effect, and two patients talked about the psychological burden to inject on a frequent basis. Overall, there was high concordance regarding emotional impact of living with a chronic disease.

Nine physicians discussed the positive psychological change following BAY 94-9027 treatment; six reported that they found the patients were happier, two said that patients were less worried, two discussed that patients were feeling better psychologically and one physician said that he found patients more confident. Twelve patients out of 16 discussed this topic; seven patients felt more confident with the new treatment, four patients reported being less worried, four patients discussed not thinking about haemophilia as often as before, four patients mentioned now having peace of mind, two patients discussed being less fearful, two patients talked about feeling better psychologically, two patients reported feeling safer with regard to preventing bleeds, one patient was less annoyed by his condition and one patient reported being calmer. Overall, there was high concordance regarding confidence gained, less worrying and being happier.

All physicians discussed the reason for change in their emotional functioning; seven reported that having fewer injections was a reason for change, five said that bleeding less frequently was one of the reasons as well, three talked about the patients being more active and three physicians said that patients had to overcome their initial uncertainty about their new treatment. All patients discussed this topic; 12 patients reported that they felt the factor lasted longer, 13 patients discussed that the longer efficacy of the product was one reason for their change in emotional functioning. Overall, there was high concordance regarding the reason for change of their emotional functioning, especially regarding factor efficacy and duration of the efficacy.

\section{Social and Work Functioning}

Eight physicians discussed the impact of the new treatment on the patients' social life and relationships; six reported no change, while two said that there was an improvement in their patients' participation in their social lives. Six patients out of the 16 discussed this topic; four patients reported no change while two patients felt more confident in engaging in social activities. Overall, there was high concordance regarding close to no change in social life.

Nine physicians discussed the impact of haemophilia on the patients' work life before switching to BAY 94-9027; two reported that 
some of their patients were missing work, two stated that some of their patients could not work, and three physicians said that some of their patients did not want to work with their disability. Eight patients out of 16 discussed this topic; four patients reported about their absence from work due to their condition, three reported that they could not work, and one patient mentioned that he did not want to inject in front of other co-workers.

Eight physicians discussed the impact of the BAY-94 treatment on the patients' work life; three reported that there was less impact on their work life, while five physicians said that there was no change. Twelve patients out of 16 discussed this topic; six patients reported that there was less impact on their work life while the other six patients reported no change. Overall, there was concordance regarding patients' relief of impact on work life, with some improvement after switching to BAY 949027.

\section{Comparing Quantitative Results with Qualitative Results}

Of the sample of patients $(n=16)$ who were interviewed, a statistically significant change $(p<0.05)$ was found on the repeated $t$ test between the baseline and end of the main study visit (week 36) on the emotional impact subscale of the Haemo-QoL-A. Patients had a higher emotional impact score at week 36 compared to baseline. This significant positive change in the emotional impact subscale supports patients' interview results shown in the previous section: patients reported feeling more confident, interacting positively with the environment, and feeling more in control.

Repeated measure $t$ tests were also computed for all the other subscales of the Haemo-QoL-A with no significant results found (Table 3 ). No significant changes in the subscales may be explained by the reality that some concepts captured by the scale may have been quite stable, whilst the concept that changed the most based on the patient interviews may not be fully explored by the scale.
Similarly, there was no significant change in the Haemo-QoL-A global score $(p=0.74)$ from baseline to the end of the main PROTECT VIII study (week 36). Since the calculation of the global score is the mean of all the subscale scores, changes may not be strong or significant enough to be reflected in the global score.

\section{DISCUSSION}

This post hoc analysis compared the perspectives of the physicians and their patients on the newly developed extended half-life product BAY 94-9027 for the prophylactic treatment of individuals with haemophilia A.

From the data extracted both groups were in general accord on the themes. Physicians and patients reported that BAY 94-9027 had no impact on the severity of bleeds but led to an improvement in the frequency of bleeds as compared to their experience with their previous treatment. These findings reflect the annualised bleeding rate (ABR) decrease observed in the PROTECT VIII trial [11]. In parallel, they reported few to no improvements regarding symptoms or physical function whilst patients were on BAY 94-9027 treatment. However, physicians and patients reported a great gain in confidence over time whilst on the treatment, as well as a gain in patient's own capacities (i.e. risk-taking). This resulted in an improvement in participation in activities (leisure, daily activities, work) after patients had overcome their initial worries about the treatment. Nevertheless, both populations reported that patients still had some restrictions due to already damaged joints. Improvement in physical functioning or mobility was not consistently reported in this 8-month study, potentially because of prophylaxis treatment prior to the study. Physicians and patients reported that it was easier to adhere to BAY 94-9027 but generally patients would have preferred an alternative to injections. On the basis of their experience in PROTECT VIII, six physicians and 11 patients spontaneously reported they would recommend the use of BAY 94-9027 for the treatment of haemophilia A. Finally, physicians and patients did not agree on potential 
Table 3 Haemo-QoL-A scores in the interviewed patient population $(n=16)$

\begin{tabular}{|c|c|c|c|}
\hline \multirow[t]{2}{*}{ Haemo-QoL-A } & \multicolumn{2}{|l|}{ Mean (SD) score } & \multirow[t]{2}{*}{$p$ value $^{\mathrm{a}}$} \\
\hline & Baseline (week 0) & End of main phase (week 36) & \\
\hline Physical functioning & $61.8(12.7)$ & $64.6(17.1)$ & 0.34 \\
\hline Emotional impact & $76.3(14.6)$ & $81.7(12.6)$ & 0.04 \\
\hline Role functioning & $79.3(16.2)$ & $79.0(13.5)$ & 0.88 \\
\hline Worry & $78.0(19.0)$ & $74.8(19.4)$ & 0.21 \\
\hline Consequence of bleeding & $78.4(20.7)$ & $74.6(23.2)$ & 0.20 \\
\hline Treatment concerns & $73.3(26.9)$ & $68.8(26.6)$ & 0.32 \\
\hline Haemo-QoL-A global score & $74.5(14.0)$ & $73.9(15.2)$ & 0.74 \\
\hline
\end{tabular}

a Paired $t$ test

treatment drawbacks, showing that they focused on different topics and priorities. This moderate overall concordance between physicians and patients suggests a good communication path between them, which is key in optimising the care, treatment and overall management of patients with haemophilia, in particular as there are several treatment options available today and more to come on the market in the near future [17].

Achieving a good level of concordance in most topics between physicians and patients, especially regarding gain in confidence, is an indicator of the strength of BAY 94-9027's impact on patients' lives. It has been shown in the literature that sometimes physicians' and patients' views regarding a treatment can be quite different and in disagreement, depending on the issues [18-20]. No statistically significant change in the Haemo-QoL-A global score was demonstrated between the baseline and end of main study assessment in the interviewed population, but a statistical significance was found in the Emotional impact subscale showing an improvement at the end of the main study compared to baseline. These quantitative results are supported by the content of the physicians' and patients' interviews.

The greatest change in patients' lives was a gain in confidence after patients were able to overcome their initial worries and were able to experience the efficacy of the new treatment.
Their newly gained confidence helped them to take more risks and be bolder, up to a limit, their main limitation being their already damaged joints.

The absence of significant changes in the Haemo-QoL-A assessment and the changes reported during the interviews could be partially explained by the time differences between the PRO completion at week 36 and the interviews which occurred after 57 months on average. Still, in an effort to fully understand the absence of significant changes in the HaemoQoL-A in other domains, in particular the physical activities, while physicians and patients had reported significant changes during the interviews, we compared the concepts raised during the interview to the concepts captured by the questionnaire. Basic activities reported by 10 patients during the interviews (e.g. picking up things, shaving, dressing, eating) were well covered by the PRO by four items (daily activity, household tasks, lifting heavy object). Similarly, mobility reported by all the patients (e.g. walking, going down stairs, limitations) was a well-covered concept in HaemoQoL-A with five items related to these concepts (e.g. walking, stairs, leaving house, traveling, geographical location of home). However, nine patients reported a wide spectrum of leisure activities and hobbies, such as running, walking for a long time, camping, various sports, or gardening. The Haemo-QoL-A asked one single 
question related to the ability to practice sports. This sole question may not be sufficient to grasp in detail what was reported by the patients. The absence of improvement in the Haemo-QoL-A between the two assessments could have been the result of high scores at baseline, limiting the ability to capture significant improvement.

This study emphasizes the added value of physician and patient interviews in clinical trials, in particular in rare diseases, to complement patient-reported outcome or clinical quantitative data and to gain additional insight into trial data, support interpretation of other quantitative assessments and endpoints to discuss meaningfulness of change [21-24].

One of the main limitations of the study relates to the representativeness of the sample of patients interviewed. The sample of 16 patients of the $110(15 \%)$ enrolled and randomized at week 10 to one of the prophylaxis treatment arms was recruited from three countries only and not from all countries participating in the study. It should be noted that the interviews were optional, and participants were free to answer or not the questions from the interviewer. Statistical tests comparing the interviewed sample with the whole study population to test for representativeness showed that the interviewed patients had experienced fewer bleeds in the 12 months before the PROTECT VIII trial. These results may be interpreted as the interview population being less clinically severe which could explain why this sample did not show a significant improvement regarding physical activities or symptoms (bleeding severity), or improvement in their social spheres (friends or family). However, their confidence was significantly improved independently of their severity which resulted in an improvement in their participation in leisure and other activities.

In addition, as the interviews were designed to collect information on BAY 94-9027, the questioning was biased towards the new treatment which could have oriented patients' answers towards treatment characteristics; this could explain the discrepancies in the PRO conceptual mapping with the interviews from patients.

\section{CONCLUSIONS}

This analysis showed that physicians and patients were in general agreement about the beneficial impact of BAY 94-9027, specifically regarding the increased level of confidence and its positive impact on patients' lives following the switch to this new treatment. Patients were generally more optimistic than clinicians regarding engagement in more activities than before and impact on work life following switch to new treatment. Qualitative findings supported the observed quantitative improvement in the Haemo-QoL-A emotional impact subscale in the pivotal PROTECT VIII trial in patients with severe haemophilia A. Patients had to overcome their initial worries regarding the treatment efficacy to fully experience its benefits. Despite only interviewing a small sample of the entire clinical trial population, based on free participation, this research provides some interesting initial insight from patients and clinicians regarding their experience of BAY 949027 upon patients' daily lives.

\section{ACKNOWLEDGEMENTS}

The authors would like to thank physicians and patients who participated in the interviews. The authors would like also to thank Sophia Kessabi, employee of Bayer at the time the project was conducted, for her contribution and scientific input.

Funding. This study as well as journal's rapid service and open access fees were funded by Bayer.

Authorship. All named authors meet the International Committee of Medical Journal Editors (ICMJE) criteria for authorship for this article, take responsibility for the integrity of the work as a whole, and have given their approval for this version to be published.

Prior Presentation. This work was presented as a poster at the ISTH 2019 Congress in Melbourne, 6-10 July 2019. 
Disclosures. Michaël Acquadro, ICON plc employee, was paid consultant to Bayer and Roche. Jérémy Lambert, ICON plc employee, was paid consultant to Bayer, Novo Nordisk and Roche. Elodie de Bock, ICON plc employee, was paid consultant to Bayer. Shadan Lalezari, has consulted/received honoraria from Bayer, Teva, Pfizer, Roche, PI Healthcare, Takeda, reimbursed for travel expenses by Alnylam, Bayer, Biogen, Biomarin, Baxalta, Grifols, Novo Nordisk, Pfizer and Roche. Mindy L Simpson has been a paid consultant for Bayer, Bioverativ/Sanofi, CSL Behring, Genentech, HEMA Biologics, Novo Nordisk, Octapharma, Takeda, and participated in speakers bureaus for Bayer and Novo Nordisk.

Compliance with Ethics Guidelines. The study was performed in accordance with the Helsinki Declaration of 1964, and its later amendments. Relevant ethical approvals were sought at the central and/or local level, as required by each recruiting clinical site. This included approvals for the conduct of this study from the following committees: Sheba Medical Center Independent Review Board (Israel), Committee of Advice nWMO-Medical Ethical Committee at Universitair Medish Centrum Groningen (Netherlands), Hagaziekenhuis local ethics committee (Netherlands), Maastricht University Medical Center-Clinical Trial Center Maastricht (CTCM) local ethics committee (Netherlands) and the Copernicus Group Independent Review Board (USA). Patients had personally signed and dated an informed consent document prior to being interviewed.

Data Availability. The datasets used and/or analysed during the current study are available from the corresponding author on reasonable request.

Open Access. This article is licensed under a Creative Commons Attribution-NonCommercial 4.0 International License, which permits any non-commercial use, sharing, adaptation, distribution and reproduction in any medium or format, as long as you give appropriate credit to the original author(s) and the source, provide a link to the Creative Commons licence, and indicate if changes were made. The images or other third party material in this article are included in the article's Creative Commons licence, unless indicated otherwise in a credit line to the material. If material is not included in the article's Creative Commons licence and your intended use is not permitted by statutory regulation or exceeds the permitted use, you will need to obtain permission directly from the copyright holder. To view a copy of this licence, visit http://creativecommons.org/licenses/by$\mathrm{nc} / 4.0 /$.

\section{REFERENCES}

1. Srivastava A, et al. Guidelines for the management of hemophilia. Haemophilia. 2013;19(1):e1-47.

2. Lieuw K. Many factor VIII products available in the treatment of hemophilia A: an embarrassment of riches? J Blood Med. 2017;8:67-73.

3. Lambert T, et al. Practical aspects of extended halflife products for the treatment of haemophilia. Ther Adv Hematol. 2018;9(9):295-308.

4. Berntorp E, et al. Advancing personalized care in hemophilia A: ten years' experience with an advanced category antihemophilic factor prepared using a plasma/albumin-free method. Biologics. 2014;8:115-27.

5. Krishnan $S$, et al. Adherence to prophylaxis is associated with better outcomes in moderate and severe haemophilia: results of a patient survey. Haemophilia. 2015;21(1):64-70.

6. Mei B, et al. Rational design of a fully active, longacting PEGylated factor VIII for hemophilia A treatment. Blood. 2010;116(2):270-9.

7. Kessler CM, et al. B-domain deleted recombinant factor VIII preparations are bioequivalent to a monoclonal antibody purified plasma-derived factor VIII concentrate: a randomized, three-way crossover study. Haemophilia. 2005;11(2):84-91.

8. Coyle TE, et al. Phase I study of BAY 94-9027, a PEGylated B-domain-deleted recombinant factor VIII with an extended half-life, in subjects with hemophilia A. J Thromb Haemost. 2014;12(4): 488-96.

9. Pipe SW, et al. Life in the shadow of a dominant partner: the FVIII-VWF association and its clinical implications for hemophilia A. Blood. 2016;128(16):2007-16. 
10. Shah A, et al. BAY 94-9027, a PEGylated recombinant factor VIII, exhibits a prolonged half-life and higher area under the curve in patients with severe haemophilia A: comprehensive pharmacokinetic assessment from clinical studies. Haemophilia. 2018;24(5):733-40.

11. Reding MT, et al. Safety and efficacy of BAY 949027, a prolonged-half-life factor VIII. J Thromb Haemost. 2017;15(3):411-9.

12. Reding MT, et al. Efficacy and safety of BAY 94-9027 is sustained for 5 years: outcomes in 33 patients in the protect VIII extension study. Blood. 2018;132(Suppl 1):2492.

13. Wells JR, et al. Exploring the impact of infusion frequency in hemophilia A: exit interviews with patients participating in BAY 94-9027 extension studies (PROTECT VIII). Patient. 2019;12(6):611-9.

14. Rentz A, et al. Cross-cultural development and psychometric evaluation of a patient-reported health-related quality of life questionnaire for adults with haemophilia. Haemophilia. 2008;14(5): 1023-34

15. Lasch KE, et al. PRO development: rigorous qualitative research as the crucial foundation. Qual Life Res. 2010;19(8):1087-96.

16. Boyatzis RE. Transforming qualitative information: thematic analysis and code development. Thousand Oaks: Sage; 1998.
17. Nossair F, Thornburg CD. The role of patient and healthcare professionals in the era of new hemophilia treatments in developed and developing countries. Therap Adv Hematol. 2018;9(8):239-49.

18. Trujols J, et al. Patient and clinician's ratings of improvement in methadone-maintained patients: differing perspectives? Harm Reduct J. 2011;8(1):23.

19. Palmer RS, et al. Substance user treatment dropout from client and clinician perspectives: a pilot study. Subst Use Misuse. 2009;44(7):1021-38.

20. Nakamura C, et al. Mining online social network data for biomedical research: a comparison of clinicians' and patients' perceptions about amyotrophic lateral sclerosis treatments. J Med Internet Res. 2012;14(3):e90.

21. Bharmal $\mathrm{M}$, et al. How to address the challenges of evaluating treatment benefits-risks in rare diseases? A convergent mixed methods approach applied within a Merkel cell carcinoma phase 2 clinical trial. Orphanet J Rare Dis. 2018;13(1):95.

22. Ervin CM, et al. Exploring the diabetic gastroparesis patient experience: patient exit interviews. Adv Ther. 2017;34(12):2680-92.

23. Schumacher KL, et al. Qualitative research contribution to a randomized clinical trial. Res Nurs Health. 2005;28(3):268-80.

24. O'Cathain A, et al. What can qualitative research do for randomised controlled trials? A systematic mapping review. BMJ Open. 2013;3(6):e002889. 